\title{
Creencias y estilos de supervisión de profesores supervisores de prácticas: Resultados en una muestra exploratoria*
}

\author{
Beliefs and Styles of Supervision of Teachers Teaching Practice Supervisors: \\ Results in Exploratory Sample
}

Crenças e estilos de supervisão de professores supervisores da prática de ensino: Mostra os resultados de um estudo exploratório

\begin{abstract}
Marcela Romero Jeldres, Vivian Alcaíno Opazo. ${ }^{a}$
a Universidad Autónoma de Chile, Facultad de Educación. Ricardo Morales 3369, Sede Santiago, Chile. Fono: 56-02- 5823000. Correos electrónicos: marcela.romero@uautonoma.cl,vivianalcaino@gmail.com
\end{abstract}

\begin{abstract}
RESUMEN
El presente artículo indaga en los estilos de supervisión predominantes y las relaciones que hay entre creencias y estilos de supervisión en el componente práctico de la Formación Inicial Docente. La investigación opta por un diseño mixto concurrente, el que por medio de cuestionarios y entrevistas, se aplica a 12 supervisores de prácticas pedagógicas en servicio. Para la reducción de la información se realiza un análisis de clúster junto con un análisis de contenido. Los resultados señalan que predomina en los supervisores de práctica un estilo colaborativo, con connotaciones directivas, no obstante, la noción de profesionalismo docente aún está desdibujada por las inconsistencias entre las creencias declaradas en su rol profesional como formador, el propio dominio de experto y el estilo de supervisión predominante.
\end{abstract}

Palabras clave: formación inicial docente, liderazgo pedagógico, prácticas pedagógicas.

\begin{abstract}
This article investigates the predominant supervision styles and the relationships between beliefs and styles of supervision in the practical component of initial teacher training. The research considers a concurrent mixed design which, by means of questionnaires and interviews, is applied to 12 supervisors of teaching practices in service. Cluster analysis and content analysis were performed to obtain specific data. The results indicate that among practice supervisors there is a dominant collaborative style, with directive connotations; however, the notion of teaching professionalism is still blurred due to inconsistencies between their beliefs on their professional role as trainers; the very domain as expert; and the main style of supervision.
\end{abstract}

Key words: initial teacher training, leadership pedagogical, pedagogical practices.

\section{RESUMO}

Investigam-se os estilos predominantes de supervisão e as relações entre crenças e estilos de supervisão no componente Prática da formação inicial de professores. A pesquisa escolhe um projeto misto concorrente e emprega questionários e entrevistas aplicados a 12 supervisores de Práticas de ensino, em serviço. Para redução de informações, é realizada a análise de agrupamento, com análise de conteúdo. Resultados indicam que domina a prática com estilo colaborativo, com conotações diretivas; no entanto, a noção de profissionalismo docente,

* Esta publicación forma parte de los resultados de investigación del Proyecto de Innovación Docente Año 2009: "Modelo de análisis colaborativo de docencia, para prácticas profesionales de EGB: Hacia la configuración de Redes escolares efectivas". Folio 027, VRA, USACH, dirigido por la Dra. Marcela Romero Jeldres. 
ainda é turva, por inconsistências entre as crenças declaradas em seu papel profissional como treinador, como o especialista de domínio e por muitos estilos predominantes de supervisão.

Palavras chave: formação de professores, liderança pedagógica, práticas pedagógicas iniciais.

\section{INTRODUCCIÓN}

La sociedad demanda de la Educación y en especial de la Formación Inicial Docente (en adelante FID), propuestas que sean capaces de generar profesionales autónomos y reflexivos de sus prácticas, de tal manera que puedan responder efectivamente a los cambios sociales que ocurren de manera vertiginosa en la sociedad del conocimiento. El currículo de la FID puede entenderse desde dos dimensiones: la formación teórica y la formación práctica. La formación teórica incluye las asignaturas asociadas a los contenidos disciplinares y pedagógicos, y la formación práctica incorpora los cursos en los que los futuros profesores tienen un acercamiento directo con el entorno escolar, ya sea a través de observaciones, como de interacción en el aula. Con todo, ambas pueden actuar estratégicamente para satisfacer las necesidades de la sociedad.

Se sostiene que las prácticas son un espacio que permite al futuro docente esclarecer aspectos vocacionales al vivenciar, en un ambiente no traumático, controlado, las experiencias docentes que estarán presentes durante su vida profesional (Contreras et al., 2010; Correa, 2011; Latorre, 2005).

Los sujetos que intervienen en el componente práctico de la FID se encuentran definidos en el concepto de tríada formativa tal como lo entiende Ávalos (2002). Estos procesos de enseñanza-aprendizaje están mediados por dos sujetos: el profesor mentor, que colabora desde el centro de prácticas, y el profesor supervisor, que lo hace desde la universidad. En ambos, así como en el profesor en formación, sus experiencias de vida, sus conceptos sobre el ser profesional e, incluso, sus interacciones configuran el sistema de creencias que afectará su toma de decisiones en el aula (Contreras, 2010; López, 2006).

El conocimiento que se tiene del profesor supervisor como formador da impulso a una investigación que permita adentrarse en la forma especial en que ellos interactúan con los profesores en formación: la supervisión, que se configura como un ámbito muy particular de interacción docente/estudiante por razones de orden variado, tales como el número de alumnos, el espacio de docencia (que no es un aula propiamente tal) y los contenidos programáticos.

En consecuencia, la supervisión de los desempeños de los profesores en formación se releva como un acto docente diferenciado, pero que, no obstante, influye muy directamente en la práctica del profesor en formación: el modo en que el profesor supervisor realiza las retroalimentaciones de los desempeños del profesor en formación, o los aspectos a los que le da más importancia se yerguen como cuestionamientos importantes al momento de afrontar a este sujeto de estudio.

Lo anterior ha llevado a cuestionarse ¿qué características tienen las creencias sobre la supervisión de los docentes del componente práctico? ¿Qué estilo de supervisión predomina en los formadores a cargo del componente práctico? ¿Qué relaciones existen entre las creencias sobre la supervisión de prácticas y los estilos de supervisión del profesor a cargo de la formación práctica de FID? Al tener en cuenta esta información, el objetivo de esta investigación busca indagar en las creencias sobre supervisión en el componente 
práctico de la FID identificando los estilos de supervisión predominantes. Específicamente, pretende identificar las creencias sobre supervisión de los docentes del componente práctico, identificar sus estilos de supervisión, y encontrar las relaciones que hay entre las creencias y los estilos de supervisión.

\section{EL COMPONENTE PRÁCTICO DE LA FORMACIÓN INICIAL DOCENTE}

La FID contempla dos ámbitos de formación: la teórica y la práctica. Respecto de la formación práctica, se considera que es un concepto de varias dimensiones, que actúa como un eje articulador entre la teoría y la práctica, y que maneja como elemento central colocar al estudiante de pedagogía en contacto con un centro educativo (Ávalos, 2002). Investigaciones señalan la formación práctica como uno de los momentos más esperados por los estudiantes de pedagogía y en la que perciben que aprenden más (Wilson, 2006).

La formación práctica posee características distintivas que la convierten en una instancia idónea de formación y de desarrollo profesional para el estudiante de pedagogía. Este proceso se estructura en la relación entre la Universidad -como centro de formación teórica y ensayo y análisis de las intervenciones prácticas- y la escuela -como ámbito de desarrollo de lo propiamente escolar y laboral-. De ahí que sea considerada un espacio de enriquecimiento de la formación identitaria y de reflexión/maduración de la tensión que, a veces en forma divergente, exigen estos dos ámbitos de formación; lo que supone en el profesor en formación desplegar sus habilidades y competencias en grados de alta complejidad y demanda de tiempos: de enfrentarse a la realidad escolar, tiempo para sopesar las tensiones internas que provoca, tiempo para la reflexión, y tiempo para la acción reflexiva.

Sabiendo que las prácticas adquieren varias formas según la unidad universitaria (Ávalos, 2002; Contreras et al., 2010; Montecinos, Barrios y Tapia, 2011; Solís et al., 2011) se reconoce que el rol del supervisor va haciéndose cada vez más presente para la orientación, reflexión y retroalimentación de los desempeños docentes. Actúa como un modelador del actuar del profesor en formación, a través básicamente del apoyo que presta desde su experticia disciplinar a los estudiantes, la que, en conciencia de sus creencias, ayuda a enriquecer la práctica docente de ambos, en la medida que así lo conceptualicen y lo realicen (Correa, 2011, 2009).

\section{LAS CREENCIAS DE LOS PROFESORES}

Las creencias dentro del pensamiento del profesor son, al igual que las concepciones y las teorías, de naturaleza implícita. Es difícil acceder a ellas sin inferirlas desde la acción. En este sentido, cobra significancia la aceptación bastante general entre los investigadores acerca de que ellas tienen una notoria influencia en el actuar (Contreras, 2010; Contreras et al., 2010; García, Azcárate y Moreno, 2006; Manterola, 2011).

Ciertamente, el autor más reconocido en la investigación sobre creencias es Manuel Pajares quien, en 1992, diseñó una definición de las creencias, considerándolas como un sistema distinto en su naturaleza al conocimiento, y más discutibles en cuanto a los elementos que la sostienen y mucho menos dinámica. Asimismo, reconoce que aunque cambien algunas, no implica que se modifique todo el sistema de creencias (Pajares, 1992). 
Por otra parte, el origen de las creencias en el pensamiento del profesor es la experiencia: ya sea afectiva, valorativa o una experiencia con el conocimiento formal (Nespor, 1987 Cit. en Da Ponte, 1999). Aun teniendo en cuenta su carácter experiencial, otros autores plantean que los aspectos que están en juego en las creencias de los docentes son: su experiencia personal, su experiencia escolar como estudiantes, y su experiencia con el conocimiento formal en comunidades de especialistas (López, 2006). Esto implica que las creencias se alimentan de elementos no reflexionados, que no tienen un sustrato teórico $-\mathrm{o}$ incluso lógico- que las explique o que se puedan relacionar.

Otra característica de las creencias es que al alimentarse las experiencias, su configuración es muy estable y pueden incluso ser obstáculos en el desarrollo profesional docente o en la formación inicial (Contreras et al., 2010). Sin embargo, la visión como obstáculo es desde los investigadores, pues las creencias en el sujeto que las pone en acción permiten organizar y afrontar la práctica, que es un espacio de incertidumbres (Manterola, 2011). De esta manera, las creencias también configuran los estilos pedagógicos, pues ayudan a simplificar la realidad con la que se interactúa (Da Ponte, 1999).

En síntesis, se puede sostener que las creencias son sistemas de experiencias de diversa índole que se nutren por la conexión que hacen en la acción docente con los conocimientos y las teorías implícitas. De otro modo, las creencias son el sustrato desde el cual se explica la acción docente, aunque el actor no siempre sea consciente de ellas.

Quienes han investigado sobre las creencias, concuerdan que tienen una fuerte influencia en la toma de decisiones en el aula, de tal manera que se puede afirmar que se actúa según la creencia. Ahora bien, puede ser que también ocurran inconsistencias entre lo que se cree que se hace y lo que efectivamente se hace en las interacciones profesor/ estudiante (Ávalos y Matus, 2010; Contreras, 2010; Contreras et al., 2010; Correa, 2009; Da Ponte, 1999; Hernández y Maquilón, 2011). Esto significa que en la práctica docente se ponen de manifiesto diferentes creencias, dependiendo del ámbito de la decisión tomada: así, el profesor puede desplegar creencias sobre evaluación, participación o conocimiento en una misma clase.

Investigaciones asociadas a este tema en Chile concuerdan en que las creencias son estables y difíciles de modificar (García y Sebastián, 2011; Leal, 2005), al tiempo que los formadores en contexto de práctica profesional adquieren una importancia vital para el éxito de la formación y en el cambio de creencias (Contreras et al., 2010; García y Sebastián, 2011; Gómez, Guerra y González, 2009).

\section{CARACTERÍSTICAS PROFESIONALES DE LOS SUPERVISORES DE PRÁCTICAS}

Los supervisores de práctica son una parte fundamental de la tríada formativa del componente práctico de la FID (Ávalos, 2003; Contreras et al., 2010; Correa, 2009; Maturana, 2011; Montecinos, Barrios y Tapia, 2011; Solís et al., 2011; Vaillant, 2002). Son considerados como formadores por cuanto se dedican profesionalmente a la tarea de formar profesores y poseen el conocimiento teórico y práctico que los faculta para ello, asî como también un compromiso con su profesión (Ávalos, 2003; Vaillant, 2002), lo que va configurando una forma específica de ser formador, basada en la experiencia de aprendizaje de nuevos saberes con los estudiantes en práctica (Correa, 2009). 
Los supervisores deben evidenciar que tienen un conocimiento del medio escolar tanto por su propia experiencia como docentes, como porque siguen ligados por otros medios a las escuelas o liceos. Junto con ello, la literatura propone que este formador debe tener una alta valoración por la actualización de sus saberes (Ávalos, 2003; Vaillant, 2002). Asimismo, su rol en el contexto de las prácticas profesionales es una instancia de desarrollo profesional personal mediante la toma de conciencia de su rol formativo (Correa, 2011, 2009; Montecinos, Barrios y Tapia, 2011; Vaillant, 2002), y de su impacto en el desarrollo profesional en el practicante a través de su experiencia, consejo, guía, apoyo, colaboración y retroalimentación (Correa, 2009), permitiendo el desarrollo de su autonomía como docente (Montecinos, Barrios y Tapia, 2011).

En países con tradiciones más arraigadas sobre la supervisión, lo que hace el supervisor y cómo lo hace ha sido una fuente constante de trabajo. En zonas como EE.UU y Canadá se han diseñado criterios para la elección de los supervisores universitarios de práctica en los que se pueden evidenciar actitudes y competencias como, por ejemplo, que estén validados como docentes de excelencia, que posean más de diez años de experiencia docente, y que posean algún posgrado o especialización docente (Correa, 2011, 2009; Gervais, 1995).

La literatura sobre la supervisión de prácticas es un ámbito investigativo poco explorado en Chile. En la síntesis de lo que se ha desarrollado hay que reconocer las investigaciones sobre la tríada formativa, en la que el supervisor es parte del estudio, aunque no el centro de mayor interés. Se ha ilustrado que el principal foco del trabajo de los supervisores de prácticas se realiza en los últimos semestres de la FID, lo que se denomina las "prácticas pre y profesionales" (Contreras et al., 2010; Montecinos, Barrios y Tapia, 2011). En ellas, los supervisores dan cuenta de sus competencias en el análisis de las acciones y en la forma en que manejan las preguntas en las reuniones, y la retroalimentación de las observaciones hechas a los profesores en formación, tanto como en la identificación de las fortalezas y debilidades que posea el practicante (Montecinos, Barrios y Tapia, 2011).

En síntesis, un formador que participa del componente práctico de la FID posee una experticia, una identidad específica, y forma parte de un grupo caracterizado por saberes particulares. Su importancia se puede expresar en que: "El supervisor aporta una mirada externa, ofrece el apoyo de la institución universitaria, establece un nexo entre los dos medios de formación, participa en la definición y la comunicación de los aprendizajes que el estudiante realiza en ese periodo formativo" (Correa, 2009: 242).

De esta manera, la supervisión de prácticas pedagógicas será entendida como una función de docencia que compromete en interacción cara a cara al supervisor con uno o un grupo de profesores practicantes, cuyo objetivo es mejorar los desempeños en las prácticas de enseñanza, bajo el supuesto de que éstas afectan el aprendizaje de los estudiantes. Con ese fin, el profesor supervisor trabaja en los ámbitos curriculares, didácticos, disciplinarios y de ética profesional del docente en formación (Correa, 2011; Gervais, 1995; MINEDUC, 2003; Montecinos, Barrios y Tapia, 2011; Sánchez, 1993).

\section{ESTILOS DE SUPERVISIÓN DE PRÁCTICAS PEDAGÓGICAS}

Teniendo en cuenta las características del trabajo del supervisor de prácticas ¿cómo desarrollan estas funciones? ¿Cuál es el estilo de supervisión que predomina en sus intervenciones? 
En el contexto de las investigaciones en Canadá, Gervais (1995) propone un esquema de estilos de supervisión, con foco en la relación entre teoría y práctica: ¿cómo se realiza esta aproximación? ¿Cuál es más predominante en el supervisor? Su propuesta destaca aspectos que el supervisor releva, tales como el comportamiento del profesor en formación, las ideas que expone o el apoyo que brinda a sus estudiantes. Estas interpretaciones que hacen los supervisores de la experiencia del practicante, en el modelo de Gervais, contemplan esencialmente las siguientes propiedades: el sentido, la orientación fundamental y lo que nos dice sobre la perspectiva de supervisión.

Con estas propiedades, las interpretaciones que hacen los supervisores de las acciones de los practicantes se pueden modelizar en tres estilos de supervisión, bajo la lógica de la transferencia desde la teoría a la práctica: un primer estilo, abierto y compartido, que integra, explora y comparte los análisis de los actos del practicante, en este caso la transferencia pasa por la reapropiación de su experiencia por el practicante. Un segundo estilo, de carácter localizado, que es un término medio en la transferencia entre teoría y práctica, que busca profundizar sobre las experiencias para generar nuevas reflexiones. Por último, un estilo determinado, en el que la interpretación de los actos busca la adquisición de elementos que permitan tener bajo control el acto docente.

En otra línea, el trabajo de Glickman (1985) en los Estados Unidos ha sido recogido por Sánchez (1994), y revalidado por las autoras en esta investigación. Se plantea, en este enfoque teórico, una definición de estilos de supervisión, en base a las creencias sobre enseñanza y aprendizaje (Sánchez, 1994). Así, se entiende que para definir cada estilo de supervisar se privilegien algunas acciones por sobre otras. Esta elección es concordante con creencias asociadas a la psicología cognitiva, a la psicología humanista y a la psicología conductista. Estas consideraciones preliminares le permitieron a Glickman establecer estilos de supervisión que se relacionan con cada una de las corrientes de psicología que se han mencionado.

Figura 1. Continuo del comportamiento del supervisor

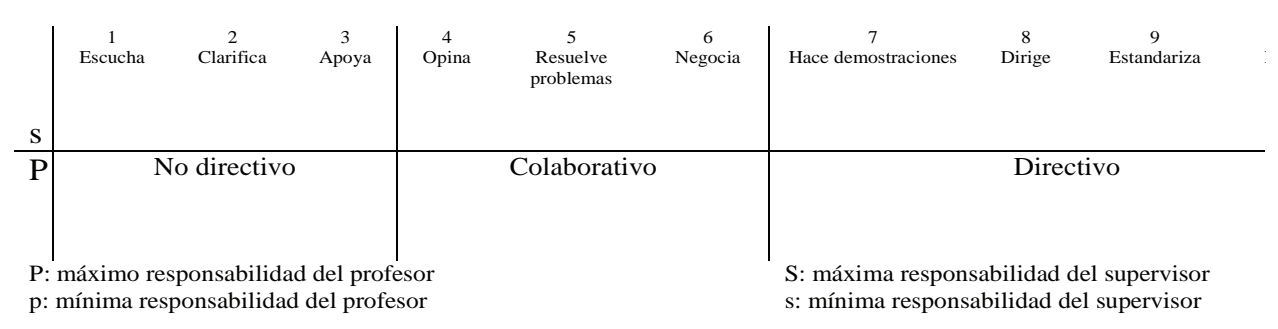

Fuente: Sánchez (1993: 113).

Estas tres orientaciones supervisoras se denominan: no directivo, colaborativo y directivo, respectivamente. Sus características se presentan en un continuo que fluctúa entre la menor y la mayor autonomía del practicante en la toma decisiones. Siguiendo las 
descripciones que Sánchez (1993) hace sobre estas orientaciones de supervisión, la Figura 1 expone las principales acciones que realiza el supervisor dentro de su estilo. Antes, sin embargo, es importante aclarar que, al igual que Gervais, el de Glickman tampoco asume que un profesor supervisor manifiesta rasgos asociables a un solo estilo de supervisión, sino que se clasifica en uno u otro, según cuáles predominen más en su acción. De la orientación directiva se puede decir que incluye comportamientos de clarificación, demostración, dirección, estandarización y reforzamiento.

El resultado final sería la realización de una tarea que el profesor hubiera llevado a cabo durante un período de tiempo determinado. Busca ser eficaz. Por lo mismo, no debe ser confundida con comportamientos arbitrarios, pues la guía de su acción es otorgarle al profesor las herramientas para que su enseñanza sea la más probadamente eficaz. Esta tendencia (directiva) parte del supuesto de que el supervisor conoce más sobre el contexto de la enseñanza y aprendizaje que el profesor, por lo tanto, las decisiones del supervisor son más efectivas que si se deja al profesor tomar las suyas propias (Sánchez, 1993: 103, 105).

La segunda orientación presentada por Glickman refiere al estilo colaborativo. La principal característica de ella es la condición de igualdad democrática que debe existir entre supervisor y practicante. Lo anterior supone una verdadera disposición al trato igualitario y también al reconocimiento de las propias limitaciones, con el fin de poder avanzar. Son propios de esta orientación los comportamientos que incluyen la escucha, aportación de ideas, solución de problemas y negociación. El resultado final vendría a ser un plan de trabajo negociado entre el supervisor y el profesor principiante en el que se delimita la estructura, el proceso y los criterios de la acción pedagógica. "La orientación colaborativa presupone que las ideas individuales sobre el proceso de enseñanza del profesor o del supervisor no son tan efectivas como si se considerasen conjuntamente" (Sánchez, 1993: 108).

Por último, en la orientación no directiva se reconoce la capacidad del practicante para tomar decisiones y hacerse cargo de los procesos, al margen de cualquier ayuda externa. En ese sentido, el rol del supervisor incluye comportamientos como el apoyo, la escucha, ser facilitador de la reflexión. "Los supervisores deben tratar de no emitir juicios ni vacilar en los deseos expresados por los profesores y además adaptar su propio comportamiento cuando los profesores demuestren desgano por generar soluciones" (Sánchez, 1993: 110).

\section{METODOLOGÍA}

Es el interés de esta investigación poder identificar los estilos predominantes en la supervisión de prácticas pedagógicas y, al mismo tiempo, se pretende abordar el fenómeno de las creencias asociadas a esta acción supervisora. Sigue la perspectiva epistemológica del pensamiento del profesor, por lo que se aloja en los estudios cualitativos, de carácter interpretativo y hermenéutico. Para poder profundizar en la tendencia de estilos y en las creencias que los definen, se optó por un diseño mixto, que entrega mayores posibilidades de enriquecer el análisis. El enfoque fue mixto concurrente (Hernández, Fernández y Baptista, 2010).

El sistema de categorías que se utilizó fue extraído por las autoras desde la teoría y condujeron la definición de los instrumentos, cada uno de los cuales abordó una categoría teórica. La Tabla 1 muestra estas categorías. 
Tabla 1. Categorías teóricas e indicadores de la categoría de los supervisores de práctica

\begin{tabular}{|l|ll|}
\hline \multicolumn{1}{|c|}{ Categorías Teóricas } & \multicolumn{1}{c|}{ Indicadores de la Categoría } \\
\hline $\begin{array}{l}\text { Características profesiona- } \\
\text { les: entendidas como aquellas } \\
\text { características que definen su } \\
\text { ser profesional y que dicen } \\
\text { relación con su preparación } \\
\text { teórica para asumir el rol a } \\
\text { cabalidad. }\end{array}$ & $\begin{array}{l}\text { Formador de profesionales de la educación (Correa, 2009; } \\
\text { Montecinos, Barrios y Tapia, 2011; Vaillant, 2002). }\end{array}$ \\
& $\begin{array}{l}\text { Perfeccionamiento para el rol (Gervais, 1995; Portelance } \text { et al., } \\
\text { 2008). }\end{array}$ \\
\hline $\begin{array}{l}\text { Actividades/acciones del su- } \\
\text { pervisor: entendidas como } \\
\text { los distintos procedimientos } \\
\text { que se desarrollan para poder } \\
\text { ejercer el rol. }\end{array}$ & $\begin{array}{l}\text { Docente de excelencia (Correa, 2009; Marcelo, 2008). } \\
\text { Dominio de experto (Correa, 2009). }\end{array}$ \\
& $\begin{array}{l}\text { Manejo de preguntas: que implica la puesta en práctica de } \\
\text { competencias asociadas a la colaboración (Portelance et al., } \\
\text { 2008), así como también un desarrollo de secuencias de } \\
\text { interacción (Montecinos, Barrios y Tapia, 2011; Waite, 2009). } \\
\text { Retroalimentación que implica acciones que informan del } \\
\text { desempeño pedagógico. Está relacionada con la evaluación, } \\
\text { estimular la reflexividad y la identificación de fortalezas y } \\
\text { debilidades (Montecinos, Barrios y Tapia, 2011; Portelance et } \\
\text { al., 2008; Schön, 1998). } \\
\text { Análisis de las acciones: que contempla el seguimiento y } \\
\text { orientación de las prácticas, consejos sobre materiales } \\
\text { didácticos, manejo disciplinar, vinculación teoría-práctica, } \\
\text { fomento del saber práctico (Correa, 2009; Molina et al., 2004). } \\
\text { Vinculación universidad-escuela (Correa, 2009; Maturana, } \\
\text { 2011; Molina et al., 2004; Romero y Maturana, 2012). }\end{array}$ \\
\hline
\end{tabular}

Fuente: elaboración propia.

\subsection{INSTRUMENTOS}

Los instrumentos seleccionados fueron: un cuestionario auto-aplicado a los supervisores de prácticas pedagógicas en ejercicio y una entrevista semi-estructurada. La construcción del cuestionario se adaptó del formato propuesto por Sánchez (1994) que utilizó en el contexto español, el cual fue validado a través del juicio de experto. Asimismo, se incorporaron los aportes destacados en la literatura que se refieren a las características profesionales y actividades/acciones del supervisor. Está constituido por tres bloques:

a) El primero busca recabar información sobre las características demográficas, de educación y de experiencia en el ámbito de la supervisión de los profesores supervisores.

b) El segundo, referido a las creencias y a los estilos de supervisión, establece 4 posibles direcciones de las preguntas, basadas en las acciones de tres estilos de supervisión definidos en la literatura: no directivo, colaborativo y directivo (Glickman, 1985). Son 53 afirmaciones organizadas en una escala Likert bajo la graduación acuerdo/desacuerdo, en la que 1 remite a 
"totalmente en desacuerdo", 2 a "en desacuerdo", 3 a "ni de acuerdo ni en desacuerdo", 4 a "de acuerdo", y 5 a "totalmente de acuerdo".

c) El tercero contempla 2 preguntas abiertas, cuyo sentido teórico aborda las características profesionales del supervisor. El grado de confiabilidad $\alpha$ de Cronbach fue determinado a partir de las varianzas, obteniendo un coeficiente de .895 lo que se considera como fiable (Hernández, Fernández y Baptista, 2010). El cuestionario fue auto-administrado, distribuido y recepcionado a través de correo electrónico. Para la construcción de este cuestionario se siguió el continuo descrito por Sánchez (1994), considerando los indicadores de actividades/ acciones de los supervisores descritos teóricamente. La Tabla 2 muestra esta relación.

Tabla 2. Pertenencia de las preguntas según dirección de las proposiciones y continuo del comportamiento del supervisor

\begin{tabular}{|c|c|c|c|c|c|c|c|c|c|c|c|}
\hline & \multicolumn{11}{|c|}{ Dirección de las proposiciones } \\
\hline \multirow{2}{*}{$\begin{array}{c}\text { Continuo } \\
\text { comportamiento } \\
\text { del supervisor }\end{array}$} & Escucha & Clarifica & Apoya & Opina & $\begin{array}{l}\text { Res } \\
\text { Prob }\end{array}$ & $\begin{array}{l}\text { elve } \\
\text { emas }\end{array}$ & Negocia & Demuestra & Dirige & Estandariza & Refuerza \\
\hline & \multicolumn{3}{|c|}{ No directivo } & \multicolumn{4}{|c|}{ Colaborativo } & \multicolumn{4}{|c|}{ Directivo } \\
\hline Dimensión: & \multicolumn{3}{|c|}{$\mathrm{ND} \rightarrow \mathrm{D}$} & \multicolumn{2}{|c|}{$\mathrm{ND} \leftarrow \mathrm{C}$} & \multicolumn{2}{|c|}{$\mathrm{C} \rightarrow \mathrm{D}$} & \multicolumn{4}{|c|}{$\mathrm{D} \rightarrow \mathrm{ND}$} \\
\hline $\begin{array}{l}\text { Actividades/ } \\
\text { Acciones del } \\
\text { supervisor }\end{array}$ & \multicolumn{3}{|c|}{$\begin{array}{l}3-10-12-13-14-20-24-25- \\
32-30-35-38-44-47-50-53\end{array}$} & \multicolumn{2}{|c|}{$\begin{array}{c}2-3-15-26- \\
33-40-48-49\end{array}$} & \multicolumn{2}{|c|}{$\begin{array}{l}6-7-9-17-22- \\
23-29-31-43\end{array}$} & \multicolumn{4}{|c|}{$\begin{array}{c}1-5-8-11-16-18-19-21-27-28-34-36-37- \\
39-41-42-45-46-51-52\end{array}$} \\
\hline Total & \multicolumn{3}{|c|}{16} & \multicolumn{2}{|c|}{8} & & 9 & \multicolumn{4}{|c|}{20} \\
\hline
\end{tabular}

Fuente: elaboración propia.

También se diseñó una entrevista de carácter semi-estructurado, que fue aplicada a una sub-muestra intencionada que permitía profundizar en las creencias sobre supervisión de prácticas pedagógicas. Esta entrevista consistió en un guion con 16 preguntas que tenían objetivos relacionados con los indicadores de características profesionales del supervisor, descritos por la literatura. Se distribuyeron de la siguiente manera: 4 preguntas permitieron recabar información de identificación y contexto del informante; 3 de las preguntas abordaron las características profesionales vinculación universidad-escuela y experiencia escolar; 6 preguntas abordaron los indicadores de estilo de supervisión y concepto de formador de profesores, y; 3 preguntas abordaron el dominio de experto y la docencia de excelencia, descritos como indicadores de las características profesionales del supervisor de prácticas pedagógicas.

La entrevista fue realizada de forma personal y separada a cada uno de los informantes, con una duración aproximada de una hora. El lugar fue escogido por el entrevistado para poder contar con una cuota mayor de comodidad (Hernández, Fernández y Baptista, 2010). Asimismo, consintió y fue informado antes de comenzar que toda la entrevista sería grabada en audio y se tomarían notas. 


\subsection{TÉCNICAS DE ANÁLISIS}

Para el análisis cuantitativo se escogió un análisis estadístico descriptivo y análisis de clúster. Los análisis descriptivos incluyeron la distribución de frecuencias. Todos los datos fueron trabajados mediante el software SPSSv17. Así, las respuestas a las preguntas cerradas del cuestionario fueron reducidas en una matriz que codificó los datos en función del continuo teórico $(1 \rightarrow 10)$ que mostró tendencias de las respuestas según estadística descriptiva. Luego fueron analizados por clúster. En el análisis cualitativo se escogió un análisis de contenido a través de las familias de datos que organiza por frecuencia el software Atlas.ti. Estos datos provinieron de las preguntas abiertas del cuestionario y de las respuestas a las entrevistas.

\subsection{MUESTRA}

La muestra es de carácter intencionada. Responde a 12 supervisores de prácticas pedagógicas que actualmente se encuentran desempeñando esta labor y que contestaron el cuestionario. Las entrevistas se realizaron a una sub-muestra de carácter intencionado que, habiendo contestado el cuestionario, poseían características percibidas como una riqueza para la interpretación de los datos y que responden a elementos que la literatura ofrece para los supervisores de prácticas (Correa, 2009). Estas características fueron la mayor experiencia como supervisor y el trabajo docente en aula.

Respecto a sus características demográficas y profesionales, se evidencia que todos los supervisores son titulados como Profesores de Estado, y de ellos el $41.7 \%$ son hombres y el 58,3\% son mujeres. En cuanto a su edad, el 41,6\% está en el rango entre 25 y 40 años, siendo la moda de toda la muestra 30 años. En lo relativo a sus características profesionales, el $75 \%$ cursa o ha obtenido el grado de Magíster, principalmente en las áreas de gestión $(41,7 \%)$ o de currículum $(41,7 \%)$. Asimismo, el $66,7 \%$ de ellos no realiza docencia en contexto escolar, sino que está abocado a la docencia universitaria. Finalmente, sus supervisiones las realizan en la enseñanza media en un 83,3\%. También destaca que en lo relativo a los años de experiencia como supervisor el promedio son cinco años, siendo el rango mínimo 1 año de experiencia y el mayor 15 años.

\section{RESULTADOS}

\subsection{ANÁLISIS DE CLÚSTER}

Con el fin de poder identificar los estilos predominantes en la supervisión de profesores en prácticas de los individuos de la muestra se realizó un análisis de conglomerado de K-medias (clúster) a través del software SPSSv17.

De esta manera, los clúster fueron organizados para identificar a los individuos miembros de cada conglomerado, según los centros de sus respuestas. Esto implica que cada individuo tendrá parte en los tres conglomerados, aunque con predominancia de uno por sobre los otros, pues sus respuestas evidencian la tendencia. Esto es coherente con lo planteado por Correa (2009), Gervais (1995), Glickman (1985) y Sánchez (1994), en cuanto a que los supervisores no creen actuar de una forma monolítica, sino que tienen rasgos de varios estilos y se adaptan al contexto. 
La Tabla 3 muestra la conformación de cada clúster y las distancias absolutas que separan los centros de respuestas del individuo en cada conglomerado. Se distingue que los tres clúster tienen características bastante homogéneas entre sí, aunque se puedan encontrar distancias mayores por cada individuo. Si se analizan los resultados en función del continuo, es interesante observar que el conglomerado 1 reúne todas las respuestas desde el 7 hasta el 10. Se infiere, por lo tanto, que aquí se agrupan las respuestas directivas del individuo. En el clúster 2, están las respuestas en el rango que incluye las opciones 6, 5 y 4, encontrándose aquí las respuestas de orden colaborativo. Finalmente, en el conglomerado 3, están todas las respuestas que el individuo dio en torno a los números 3, 2 y 1.

Tabla 3. Pertenencia al clúster según centros de respuesta y distancias entre centros en cada conglomerado

\begin{tabular}{|c|c|c|c|c|c|c|}
\hline \multirow{3}{*}{ Individuos } & \multicolumn{3}{|c|}{ CLUSTER } & \multicolumn{3}{|c|}{ Distancias (absolutas) } \\
\hline & Directivo & Colaborativo & No Directivo & \multirow{2}{*}{$3-1$} & \multirow{2}{*}{$2-1$} & \multirow{2}{*}{$3-2$} \\
\hline & 3 & 2 & 1 & & & \\
\hline E_1 & 8,04 & 4,75 & 1,40 & 6,64 & 3,35 & 3,29 \\
\hline E_2 & 7,88 & 5,10 & 1,60 & 6,28 & 3,50 & 2,78 \\
\hline E_3 & 7,48 & 5,00 & 3,40 & 4,08 & 1,60 & 2,48 \\
\hline E_4 & 8,08 & 4,10 & 1,60 & 6,48 & 2,50 & 3,98 \\
\hline E_5 & 7,72 & 4,55 & 1,60 & 6,12 & 2,95 & 3,17 \\
\hline E_6 & 8,12 & 4,65 & 2,00 & 6,12 & 2,65 & 3,47 \\
\hline E_7 & 7,12 & 5,20 & 1,80 & 5,32 & 3,40 & 1,92 \\
\hline E_8 & 6,72 & 4,90 & 2,80 & 3,92 & 2,10 & 1,82 \\
\hline E_9 & 8,68 & 4,30 & 1,40 & 7,28 & 2,90 & 4,38 \\
\hline E_10 & 7,28 & 4,60 & 3,00 & 4,28 & 1,60 & 2,68 \\
\hline E_11 & 8,08 & 4,50 & 1,40 & 6,68 & 3,10 & 3,58 \\
\hline E_12 & 8,40 & 4,40 & 1,40 & 7,00 & 3,00 & 4,00 \\
\hline
\end{tabular}

Fuente: elaboración propia.

De estas respuestas, en el clúster 3, mientras más cercanas a 10 indica que las acciones que el supervisor declara hacer son de estilo directivo; en el clúster 2, mientras más cercanas a 6, sus acciones tienden a lo directivo y, al contrario, si son más cercanas a 4 tienden hacia el estilo no directivo. Por último, las respuestas que se ubican más cerca del 1 , son claramente no directivas, de tal modo que la toma de decisiones recae directamente en el profesor en formación.

Respecto del clúster 3: Estilo Directivo, su rol es preponderante en la interacción, pues la lidera y la modela. Los profesores realizan acciones como demostrar -haciendo- cuáles son las mejores estrategias para resolver situaciones de aula y dirigen las intervenciones hacia los objetivos que tiene previsto o hacia las soluciones que ya contempló para el profesor en práctica. Respecto del clúster 2: Estilo Colaborativo, las respuestas de los individuos se organizan en torno a un promedio de 4,67. Esto significa que los profesores supervisores apoyan la tarea de los practicantes, y en gran medida, tienden a tener una participación más activa en la resolución de los problemas que se presentan. El sentido 
de esta colaboración sigue siendo, en la mayoría de los individuos encuestados, hacia el estilo directivo. Finalmente, en el clúster 1: Estilo No Directivo, se clasifican las respuestas declaradas como no directivas por los supervisores. Las acciones que llevan a cabo bajo esta lógica, se centran en el apoyo que brindan a los profesores en práctica. Este apoyo, es tanto en la toma de decisiones directamente asociadas a la enseñanza, como un soporte emocional. Por lo mismo, hay un factor importante de escucha en sus acciones.

Profundizando en estas descripciones, se analizan los elementos diferenciadores entre los individuos y que refleja el estilo predominante de sus acciones de interacción supervisora. Al analizar las distancias que hay entre las respuestas de los distintos clúster, se perciben más claramente las creencias que hay en el estilo de supervisar.

Figura 2. Diferencia absoluta en estilos de supervisión según distancia entre centros de clúster por individuo

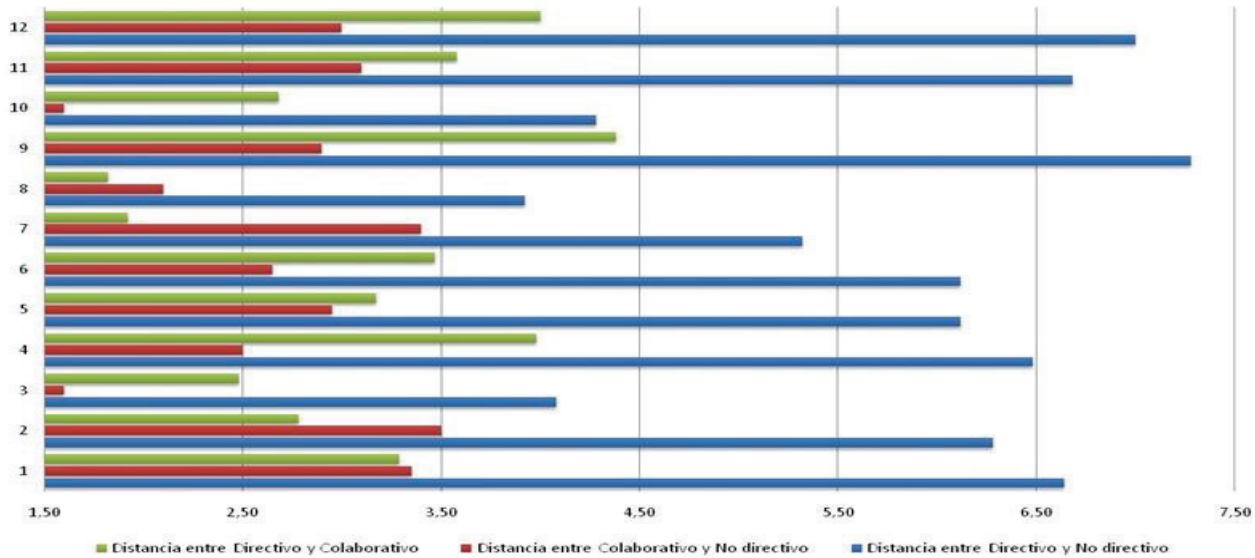

Fuente: elaboración propia.

La Figura 2 presenta en conjunto las distancias que hay entre el clúster 1 y el 2, entre el 2 y el 3 , y entre el 1 y el 3. La mayor distancia significa que la acción es expresiva de un estilo en particular, de modo que cuando se actúa en estilo directivo, la distancia aumenta respecto del estilo no directivo.

En cuanto a los estilos predominantes entre los supervisores, estas distancias muestran con mayor claridad las acciones colaborativas que realizan los profesores supervisores, por lo que sirve para poder ubicar los principales actos que corresponden con este estilo. Así, entre los individuos E3, E8 y E10 se refleja un estilo colaborativo, pues sus distancias en conjunto son las menores. Al contrario, los individuos E1, E2, E9, E11 y E12 tienen una clara tendencia directiva, con características dicotómicas, pues no refieren con claridad acciones colaborativas, pero declaran actuar en forma no directiva. En otras palabras, actúan escuchando y apoyando, pero opinan y negocian poco, y ante todo dirigen, modelan o refuerzan las acciones de los profesores en práctica. 


\subsection{ANÁLISIS DE CONTENIDO}

En términos teóricos el supervisor de práctica puede ser conocido en dos dimensiones: desde la perspectiva profesional, en la que pone en juego su autoconcepto, su experiencia como docente y los aspectos referidos a la excelencia docente y a los perfeccionamientos. Y desde la dimensión de sus acciones: en ese caso se contempla la retroalimentación, el análisis de las acciones y el manejo de preguntas.

En función de facilitar la comprensión de estas creencias, se ha seguido la misma posición teórica que permitió identificar los estilos: el continuo entre comportamientos directivos, colaborativos y no directivos. No obstante, se releva en las creencias el sentido dialéctico que entrega la enseñanza, a saber: si el rol principal lo ejerce el docente o lo ejerce el estudiante. O, como se infiere del estilo colaborativo de supervisión, es una relación en términos de igualdad. Buscando entre los supervisores, desde la literatura, los aspectos que forman el "ser profesional", se pesquisó la siguiente asignación de importancia derivada de la frecuencia con la que la categoría se iba estructurando.

Figura 3. Características profesionales que intervienen en el rol de supervisor

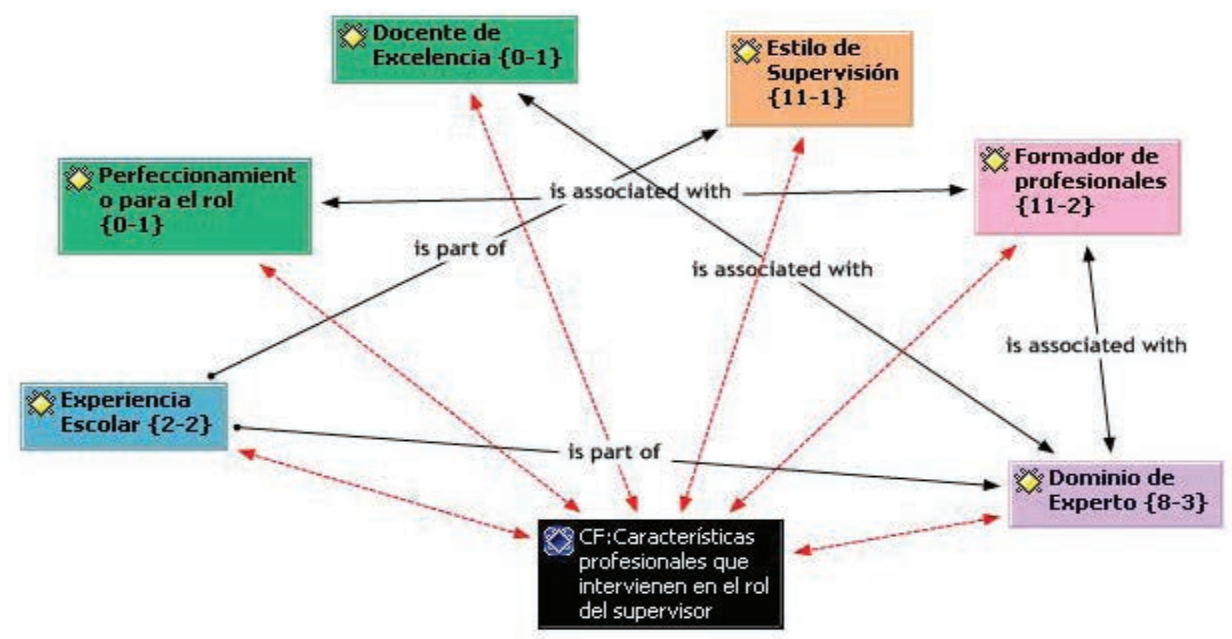

Fuente: elaboración propia.

Bajo este principio es posible sostener que, entre los supervisores consultados, aparece con mucha insistencia el estilo (Ver Figura 3). Este estilo implica creer en la co-evaluación entre los profesores en formación como vía de aprendizaje, y en la valoración de los tratos cordiales. En lo referido a su rol formativo, se distinguen creencias bastante amplias: el rol formador es tanto de componente afectivo, como técnico.

Llama la atención de las declaraciones de los supervisores, tanto en la entrevista como en el cuestionario, que su interacción bajo la lógica de dominio de experto se basa en aspectos afectivos y actitudinales. En varias de las respuestas emerge una creencia de que 
el trabajo de formación de profesores está poco esclarecido: que es necesario ponerse de acuerdo en los focos de análisis; que son necesarias las reuniones entre los supervisores para saber cómo realiza su trabajo el resto del equipo de supervisores; que se deben aunar los criterios de evaluación y la rigurosidad en el detalle. Así, se descubre en sus declaraciones el esfuerzo dialéctico de conjugar la creatividad y el estilo personal, con estándares válidos para todos los docentes.

Sin embargo, a las categorías docente de excelencia y el perfeccionamiento para el rol, les asignan una baja frecuencia en sus menciones, por lo que se puede inferir que en las creencias profesionales que tienen los supervisores de su rol, no está presente la idea de actualizar los conocimientos y de buscar perfeccionamientos que apunten a mejorar sus prácticas docentes en esta situación en particular, lo que redundaría en que los esfuerzos que llevan a cabo, y que se describieron en el párrafo anterior, serían más efectivos.

Un análisis parecido se puede hacer respecto de la categoría de experiencia escolar, entendida como la conexión con el mundo escolar a través fundamentalmente de la docencia. Desde ese punto de vista, las creencias profesionales todavía apuntan hacia actuaciones que colocan al supervisor en una situación de estatus sobre los profesores en formación: estatus que se legitima en la supervisión por parte de los mismos profesores en formación, quienes siguen las indicaciones hechas por el supervisor especialmente en las primeras intervenciones. Esto es coherente con las expectativas de autoeficacia que ellos poseen y que fueron descritas por Montecinos, Barrios y Tapia (2011).

Figura 4. Características profesionales/cambios al rol de supervisor

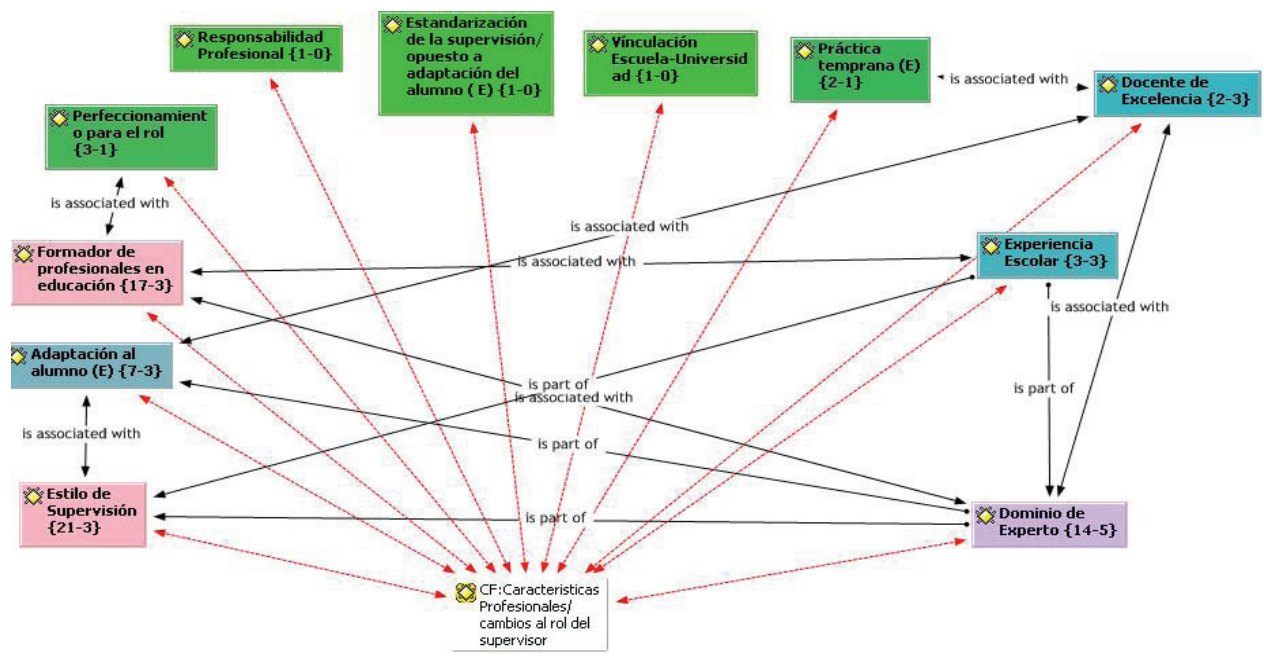

Fuente: elaboración propia.

Lo anterior se confirma al analizar el esquema de relaciones que se declaran de las características profesionales y de organización que, desde su punto de vista, deben ser 
cambiadas para generar mejoras (Ver Figura 4). En ella refieren aspectos emergentes, pero que son importantes en esta relación, tales como la adaptación al alumno, las prácticas tempranas y la responsabilidad profesional. En la primera, se declaran en favor de atender las particularidades de los profesores en formación con mucha más dedicación para poder superar sus dificultades y atender más a sus talentos. En la misma línea están las declaraciones a favor de las prácticas tempranas.

Por último, emergió la categoría de responsabilidad profesional en la pregunta acerca de las mejoras que implementaría en el proceso de prácticas. Es una categoría con sentido negativo, pues se concibe la acción de mejora desde el otro y no desde el propio supervisor, lo que indica una creencia en la que se le resta importancia formativa al componente práctico; en definitiva, se declara la importancia formativa de la práctica pedagógica y del rol del supervisor para que así sea, pero también se declara que la base de la formación viene de la teoría. Se mantienen las creencias que suponen que la práctica profesional es el espacio en el que el profesor en formación vierte todo lo que ha aprendido en sus años previos de formación (Ávalos, 2002; Correa, 2011).

Estas categorías dan cuenta de una visión de la formación práctica bastante amplia, pero no necesariamente integrada. Esto porque sólo la categoría de adaptación al alumno se relaciona fuertemente con los otros elementos que componen el esquema: se encuentra asociada a la docencia de excelencia, que aparece mejor aspectada respecto de los otros análisis. Esto se explica porque en este esquema se incorporan las respuestas de todos los participantes de la investigación, entre las que hay personas con estilos colaborativos.

\subsection{TRIANGULACIÓN: ANÁLISIS MIXTO}

Se presentan los estilos de supervisión predominantes en los individuos de la muestra, mediante la exposición de sus centroides de respuesta en cada clúster. Es decir, que a cada clúster se definió su centroide y a partir de ese valor se pudo determinar el centroide de todos ellos. Esto es, un valor que refleja exactamente en dónde se ubican los centros de las respuestas: en este caso fue de 6,77, lo que equivale a presentar que el estilo predominante de todos los individuos de la muestra es el estilo colaborativo con una fuerte tendencia hacia lo directivo, lo que implica que realizan acciones mayoritariamente de negociación, pero también de modelización de las acciones que se consideran más efectivas. El rol del supervisor queda en una posición de mayor presencia que la del profesor en práctica; de ello se puede argüir que la negociación no es en igualdad de condiciones (democrática e igualitaria), sino que se ejerce la autoridad que da el rol de profesor para poder dirigir las acciones. Se atenúa esta tendencia con acciones que tiendan a buscar el consenso del profesor en práctica.

Además se pudo determinar que, en esta misma tendencia, hay supervisores que son más colaborativos directivos, y que hay otros que son claramente directivos. Llama la atención, no obstante, que no se encontraran personas con estilos no directivos, aunque muestren una disposición muy favorable en sus respuestas.

En la Figura 5, con azul se representa a los individuos cuyos centroides de los 3 clúster se ubican sobre el valor de corte 6,77 , y en verde, aquéllos que están más abajo. Entre los primeros están quienes poseen un estilo directivo (E1, E4), y los que poseen un estilo colaborativo con marcada tendencia directiva (E2, E5, E6, E9, E11 y E12). Entre los segundos están quienes tienen un estilo colaborativo (E3, E7, E8, E10). 
Figura 5. Centroides de los 3 clústers por individuos

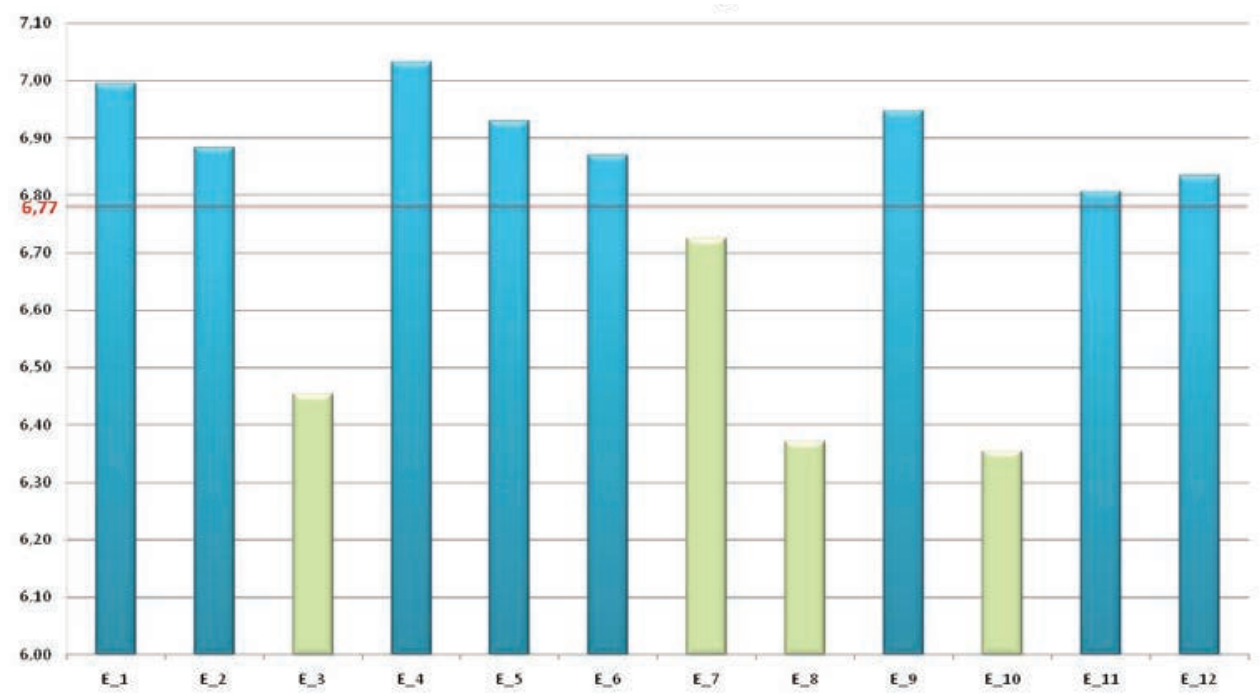

Fuente: elaboración propia.

De esta manera, al analizar a los sujetos más paradigmáticos del estilo colaborativo: E3, E7, E8 y E10, sus centroides de respuesta se ubican en el rango entre 6,30 y 6,50, excepto E7 que tiene el centroide de respuesta más alto (levemente sobre 6,70).

Esto también coincide con sus datos en las respuestas del clúster 3: estos sujetos obtienen los centroides más bajos. Al contrastar esta información con las declaraciones emitidas, se aprecia que existe una congruencia bastante alta. Por ejemplo, al ser consultados por cambios que harían al proceso de prácticas, sus respuestas ahondaron en aspectos como la reflexión, el acompañamiento, la interrelación entre teoría y práctica que debe haber en su trabajo, y la ayuda a conformar un estilo docente propio en el profesor en formación, siendo facilitador de herramientas pedagógicas.

Todas ellas evidencian una creencia acerca de la supervisión como una instancia de desarrollo profesional para el profesor en formación (potenciar sus capacidades y ayudar a configurar un estilo docente a través de la reflexión), y también de él mismo, mediante la reflexión de aspectos teóricos y prácticos que intervienen. Es deseable que en este caso se profundice en cómo se llevan a cabo estas acciones, a la luz de las dimensiones descritas por Gervais (1995) sobre la relación teoría y práctica en el supervisor universitario.

En el caso de los individuos que se encontraban, por sus centroides de respuesta totales, en el estilo directivo (E1, E2, E4, E5, E6, E9, E11, E12) manifiestan estar de acuerdo con proposiciones que sugieren actos de demostración y dirección de la interacción, principalmente. Esto se obtiene porque los centroides de respuesta se ubican sobre el valor de corte, pero sin superar el valor 7,10 ${ }^{1}$. Al igual que en el caso de las personas con estilos colaborativos, la coherencia entre los datos arrojados por el centroide de los 3 clúster por individuo

Ver Figura 5 Centroides de los 3 clúster por individuos. 
se relacionan positivamente con las declaraciones emitidas en las entrevistas y en el cuestionario. Asimismo, se destaca que las personas que contestaron la entrevista se relaciona positivamente, según sus declaraciones y sus grados de acuerdo, con las afirmaciones en el estilo directivo.

Las respuestas de estos participantes en cuanto al rol del supervisor en el proceso de prácticas, abordan temas como ser modelo y guía para la acción docente del profesor en formación, trabajo riguroso en lo técnico, y un acento en aspectos que denominan profesionales, aunque no explicitan cuáles serían. Ciertamente, en todos ellos se desprende una activa participación del supervisor como conductor del proceso, lo que en definitiva es la característica más sobresaliente del estilo.

La excepción a esta relación positiva fueron los individuos E1, E4 y E9 quienes poseen, según el centroide de las respuestas, los valores más altos. Sin embargo, en sus respuestas abordan temas como la ayuda al profesor en formación para potenciar sus habilidades, la estrategia socrática como vehículo de interacción, y la mediación y facilitación de oportunidades para el desarrollo integral del profesor en formación. Estas respuestas son propias de un estilo colaborativo, que implica la noción de un trabajo en conjunto como la vía más certera para el aprendizaje profesional de los profesores involucrados.

Lo anterior implica que hay incoherencia entre lo que se dice que hace y lo que se cree que se hace. Una forma de interpretar esta inconsistencia remite a la falta de autorreflexión de sus prácticas docentes y de retroalimentación con los profesores en formación con los que trabajan; desde otro punto de vista, la incoherencia pueda reflejar un dominio de experto débil en la relación teoría y práctica, aspecto que también estaría relacionado con el desarrollo de su ser profesional como supervisor. En todo caso, este hallazgo queda planteado para ser estudiado con mayor profundidad en futuras investigaciones.

\subsection{DISCUSIÓN}

Desde el componente práctico de la FID estos resultados parecen estar en consonancia con lo planteado por las investigaciones. En ese sentido, el supervisor tiene un rol formador actuando desde su experticia disciplinar. De este modo, el concepto que tienen de sí mismos los supervisores está en consonancia con lo planteado por Maturana (2011) respecto a la percepción que los estudiantes de pedagogía tienen del rol del supervisor en las prácticas. En ambos casos se relevan adjetivos como formador o mentor emocional. Y en ambas investigaciones se considera el factor afectivo y emocional -propio de las creencias- como base para la legitimación del rol, especialmente del dominio de experto.

De la misma forma que Esteve (2009), las investigaciones recogidas para el contexto canadiense apuntan a hacerse cargo de los elementos -competencias en su lenguajeque configuran la acción profesional del profesor supervisor (Portelance et al., 2008). En el caso de esta investigación los resultados arrojan que la noción de profesionalismo docente aún está desdibujada, fundamentalmente porque está impregnada de experiencias cotidianas, que son analizadas como experiencias de aprendizaje para ser profesor en las retroalimentaciones.

Se aprecia, entonces, que las creencias operan en este autoconcepto profesional más que en los conocimientos. Es decir, frente a la existencia de instrumentos validados y de uso público -como el MBE (MINEDUC, 2003)- e investigaciones que se acercan con rigurosidad a las prácticas pedagógicas con foco en la reflexión en la acción (Montecinos, Barrios y Tapia, 
2011) se refleja, por parte de los entrevistados y del grupo muestral, un desconocimiento que también puede ser interpretado como una escasa profesionalización de su rol.

En relación a la categoría experiencia escolar, las investigaciones apuntan a que la sostenida experiencia del supervisor en los centros escolares es una de las fuentes primordiales que hacen de su supervisión una práctica efectiva. Correa (2009) señala al respecto que, en promedio, 15 años de docencia en contexto escolar hacen posible la conjunción teoría y práctica que requiere un supervisor eficaz. Sin embargo, los resultados de esta investigación van en la línea contraria, más del $80 \%$ de los supervisores no está en contacto con la realidad escolar y su docencia la realiza en la Educación Superior.

Los comportamientos colaborativos con tendencia directiva que se obtuvieron en el estudio entran en contradicción con los resultados obtenidos por Sánchez (1993) en relación al contexto español, quien obtuvo mayoritariamente estilos no directivos. Atenúa esta situación, el que la autora aplicó su instrumento a profesores supervisores que estaban asistiendo a un seminario sobre la estrategia de supervisión clínica, por lo que ya habían comenzado una lógica de reflexión sobre la práctica que, por lo exploratorio de esta investigación, en el plano chileno recién se inicia.

\section{CONCLUSIONES}

Actualmente, conocer sobre las creencias que sostienen los docentes respecto a las dimensiones específicas de su rol se ha convertido en una necesidad que promueve la reflexión sobre la práctica y, por consiguiente, una mayor consciencia del rol formativo que posee. Y en este contexto, los supervisores de prácticas pedagógicas no quedan fuera de esta realidad.

El análisis mixto lleva a la conclusión de que existe una coherencia entre lo que cree y lo que cree que hace el supervisor de prácticas en la mayoría de los casos (75\%), emergiendo creencias asociadas a una mayor figuración del docente supervisor por sobre el profesor en formación. En la misma línea, se concluye que los profesores en formación lideran las interacciones, cuando éstas se refieren a situaciones cotidianas y experiencias vividas. Aun cuando entre los supervisores predomina un estilo colaborativo, la supervisión de prácticas adquiere connotaciones directivas, puesto que se pone en el centro de las interacciones al docente y no al profesor en formación, fuertemente avalado por la creencia de dominio de experto que se sostiene.

Finalmente, emergen inconsistencias entre las creencias declaradas en su rol profesional como formador y dominio de experto y el estilo de supervisión predominante en sus respuestas al cuestionario en el $25 \%$ de los sujetos. Esta situación se vislumbra como una arista nueva para futuras investigaciones. Es posible, también, proyectar los instrumentos utilizados y validados, así como el levantamiento teórico que se hizo en nuevas investigaciones para el contexto nacional de supervisión de prácticas pedagógicas.

\section{REFERENCIAS BIBLIOGRÁFICAS}

Ávalos, B. y Matus, C. (2010). La Formación Inicial Docente en Chile desde una óptica internacional. Informe Nacional del Estudio Internacional IEA TEDS-M. Santiago de Chile: Ministerio de Educación. 
(2003). La Formación inicial docente en Chile. Recuperado de http://www.dfpd.edu.uy/cfe/ institucional/comisiones/autoevaluacion/documentos/for_doc_chile.pdf, consultado en mayo de 2011. (2002). Profesores para Chile, historia de un proyecto. Santiago: MINEDUC.

Contreras, I., Ritterhaussen, S., Montecinos, C., Solís, M. C., Núñez, C. y Walker, H. (2010). La escuela como espacio para aprender a enseñar: Visiones desde los programas de formación de profesores de educación media. Estudios Pedagógicos, vol. XXXVI, n. 1, 85-105.

Contreras, S. (2010). Las creencias y actuaciones curriculares de los profesores de ciencias de secundaria de Chile. Tesis Doctoral, Departamento de Didáctica de las Ciencias Experimentales, Universidad Complutense de Madrid, España.

Correa, E. (2011). La práctica docente: Una instancia de desarrollo profesional. Perspectiva Educacional, vol. 50, n. 2, 77-95. (2009). El supervisor de prácticas: Recursos para una supervisón eficaz. Pensamiento Educativo, vol. 44-45, 237-254.

Da Ponte, P. (1999). Las creencias y concepciones de maestros como un tema fundamental en la formación de maestros. Traducción de ponencia publicada en On Research in Teacher Education: From a Study of Teaching Practices to Issues in Teacher Education (pp. 43-50). Recuperado de http:// www.educ.fc.ul.pt/docentes/jponte/DOCS-SP/Las\%20creencias.pdf, consultado en mayo de 2011.

Esteve, M. (2009). La formación de profesores: Bases teóricas para el desarrollo de programas de formación inicial. Revista de Educación, n. 350, 15-29.

García, L., Azcárate, C. y Moreno, M. (2006). Creencias, concepciones y conocimiento profesional de profesores que enseñan cálculo diferencial a estudiantes de ciencias económicas. RELIME, vol. 9, n. 1, 85-116.

García, M. R. y Sebastián, C. (2011). Creencias epistemológicas de estudiantes de pedagogía en educación parvularia, básica y media ¿Diferencias en la Formación Inicial Docente? PSYKHE, vol. 20 , n. 1, 29-43.

Gervais, F. (1995). Superviseurs universitaires et formation pratique en milieu scolaire: Orientation de la médiation entre théorie et pratique. Revue des Sciences de l'Education, vol. 21, $\mathrm{n}$. 3, 541-560. Bacon.

Glickman, C. (1985). Supervision of Instruction: A Developmental Approach. Boston: Allyn \&

Gómez, V., Guerra, P. y González, M. (2009). Explorando el cambio epistemológico y conceptual en la Formación Inicial de Profesores en distintos contextos universitarios. Proyecto

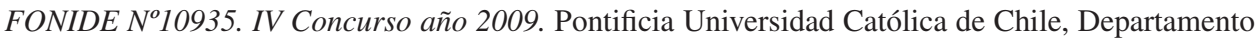
de Presupuesto y Planificación, Santiago de Chile.

Hernández, R., Fernández, C. y Baptista, M. D. (2010). Metodología de la Investigación (5 ${ }^{\text {ta }}$ Ed.). México D.F.: McGraw-Hill.

Hernández, S. y Maquilón, J. (2011). Las creencias y concepciones. Perspectivas complementarias. REIFOP, vol. 14, n. 1, 165-175.

Latorre, M. (2005). Continuidades y rupturas entre formación inicial y ejercicio profesional docente. Revista Iberoamericana de Educación, vol. 36, n. 2, 1-12.

Leal, F. (2005). El efecto de la formación inicial docente en las creencias epistemológicas. Revista Iberoamericana de Educación, vol. 34, n. 5, 1-17.

López, G. (2006). Ser maestro en el bachillerato: Creencias, identidades y discursos de maestros en torno a las prácticas de literacidad. Perfiles Educativos, vol. 28, n. 112, 40-67.

Manterola, C. (2011). Lo que piensan de la enseñanza y lo que hacen los profesores universitarios. Ensaio Pesquisa em Educacao em Ciências, vol. 13, n. 1, 139-155.

Marcelo, C. (2008). El profesorado principiante. Inserción a la docencia. Barcelona: Octaedro.

Maturana, D. (2011). Relaciones dialógicas entre la triada formativa y la inserción en aula: Hacia la propuesta de un modelo de práctica para la Formación Inicial de Profesores de Educación General Básica. Tesis de Maestría, Departamento de Educación, Universidad de Santiago de Chile. 
MINEDUC. (2003). Marco para la Buena Enseñanza. Recuperado de http://www.rmm.cl/ usuarios/equiposite/doc/200312031457060.mbe.pdf, consultado en octubre de 2011.

Molina, E., Bolívar, A., Burgos, A., Domingo, J., Fernández, M., Gallego, Mª J., Iranzo, P., León, M ${ }^{\mathrm{a}}$ J., López, $\mathrm{M}^{\mathrm{a}}$ C., Molina, M ${ }^{\mathrm{a}}$ A., Pérez, P. y Ponce, C. (2004). Formación práctica de los estudiantes de pedagogía en las universidades españolas. Profesorado. Revista de Currículum y Formación del Profesorado, vol. 8, n. 2, 1-24.

Montecinos, C., Barrios, C. y Tapia, M. F. (2011). Relación entre estilos de supervisión durante la práctica profesional y las creencias de autoeficacia de los estudiantes de Pedagogía en Educación Básica. Perspectiva Educacional, vol. 50, n. 2, 96-122.

Pajares, F. (1992). Teacher's Beliefs and Educational Research: Cleaning Up a Messy Construct. Review of Educational Research, vol. 62, n. 3, 307-332.

Portelance, L., Gervais, C., Lessard, M. et Beaulieu, P. (2008). La formation des einsegnants associés et des supervisoires universitaires: Cadre de reference. Quèbec: Ministère de l'Éducation, du Loisir et du Sport.

Romero, J., M. y Maturana, C. D. (2012). La supervisión de prácticas pedagógicas: ¿Cómo fortalecer la triada formativa? Magis, Revista Internacional de Investigación en Educación, vol. 4, n. 9. 653-667.

Sánchez, M. R. (1994). Inventario de creencias del supervisor (I.C.S.): Un instrumento para estudiar los diferentes estilos de supervisión. Enseñanza: Anuario Interuniversitario de Didáctica, vol. XII, 109-137.

(1993). La supervisión clínica como estrategia de formación de profesores mentores y principiantes. Sevilla: Universidad de Sevilla.

Schön, D. (1998). El profesional reflexivo: Cómo piensan los profesionales cuando actúan. Barcelona: Paidós.

Solís, M. C., Núñez, C., Contreras, I., Rittershaussen, S., Montecinos, C. y Walker, H. (2011). Condiciones de la formación práctica de los futuros profesores. Estudios Pedagógicos, vol. XXXVII, n. 1, 127-147.

Vaillant, D. (2002). Formación de formadores. Estado de la práctica. PREAL, n. 25, 2-47.

Waite, D. (2009). No es cuestión de datos. La evolución de un currículo de supervisión clínica y la pedagogía. Educar, n. 44, 67-78.

Wilson, E. (2006). The Impact of Alternative Model of Student Teacher Supervision: View of Participants. Teaching and Teacher Education, n. 22, 22-31. 